\title{
Spectral properties and pseudogaps in a model with d-wave pairing symmetry
}

\author{
Bumsoo Kyung \\ Département de physique and Centre de recherche en physique du solide. \\ Université de Sherbrooke, Sherbrooke, Québec, Canada J1K $2 R 1$
}

(March 30, 2000)

\begin{abstract}
A model with d-wave pairing symmetry is studied by employing a non-perturbative sum rule approach. At low temperature the magnitude of a normal state pseudogap shows strong $\vec{k}$ or angle dependence well fitted by $\cos 2 \phi$ form. With increasing temperature, the pseudogap closes at some critical angle $\phi_{c}$ and beyond this angle a single quasiparticle-like peak appears. The resulting Fermi surface is strongly temperature dependent. Both in the spectral function and the density of states, the pseudogap disappears in a manner that the spectral weight fills in the pseudogap instead of closing it with increasing temperature. All these features are qualitatively consistent with ARPES for underdoped cuprates.
\end{abstract}

PACS numbers: 71.10.Fd, 71.27.+a

The nature of an excitation gap in high-temperature superconductors has been one of the puzzling issues in the community of condensed matter physicists. Through several years of extensive experimental work, general consensus regarding the superconducting gap symmetry seems to be reached that the superconducting gap has mainly d-wave character with possibility of a small mixture of other angular momentum states, [1] 3] in contrast to conventional BCS superconductors with an isotropic s-wave gap. Recent discovery of a normal state pseudogap in underdoped cuprates has shed another side of the anomalous behaviors in the copper oxide superconductors. For these materials the low frequency spectral weight begins to be strongly suppressed below some characteristic temperature $T^{*}$ higher than $T_{c}$. This behavior has been observed through various experimental probes such as photoemission, [4 6] specific heat, [7] tunneling, [8] NMR, [9] and optical conductivity. 10] In particular recent angle resolved photoemission spectroscopy (ARPES) [4 6] and tunneling experiments 8 indicate that the pseudogap phenomenon is closely related to pairing fluctuations. These measurements clearly exhibit that the normal state pseudogap has the same angular dependence and magnitude as the superconducting gap and that often the only difference between the spectra in the pseudogap state and the superconducting state is in their linewidths. Typically $T^{*}$ is much higher than $T_{c}$ and their doping dependence is qualitatively different. While $T_{c}$ decreases with underdoping, $T^{*}$ increases in contrast. This feature suggests that $T^{*}$ does not follow $T_{c}$ characterized by long-range phase coherence, but instead some kind of a mean-field critical temperature $T_{M F}$. In spite of several possible scenarios such as the spinon pair formation without the Bose-Einstein condensation of holons, 11 13. strong superconducting phase fluctuations [14 16] and a magnetic scenario near the antiferromagnetic instability 17,18 and so on, at present there is no consensus in the origin of the pseudogap.
For the past several years extensive theoretical effort has been also made by several groups to understand this anomalous pseudogap behavior in the context of short range effective (attractive) interaction between electrons. This may be divided into two different classes of approach. In the first class, quantum Monte Carlo (QMC) simulations were made for the attractive Hubbard model. 19 26] Although this Hamiltonian is not a realistic model for understanding the complex physical behaviors in the underdoped cuprates, it is believed to capture the important ingredient of the paring fluctuations in those materials. In the absence of a small parameter this numerical method has played an important role in understanding of the model, in spite of some uncertainties due to finite size effect and numerical analytical continuation. In this approach the one-particle spectral function as well as the density of states clearly show the precursor of the superconducting gap in the normal state. In the second class, various assumptions and approximations are used. This class includes the effect of vortex phase fluctuations on the single-particle properties [16] and the 'paring approximation' theory, 27] and Tmatrix and self-consistent T-matrix approximations for the two-dimensional attractive Hubbard model. 28 33] More recently the self-consistent T-matrix approach was also applied to a model with $d_{x^{2}-y^{2}}$ pairing. 34,35] The present approach is a variant of the T-matrix approximation applied to a model with d-wave pairing symmetry.

In a previous study, 36] a non-perturbative sum rule approach was developed for the attractive Hubbard model by extending previous work on the repulsive Hubbard model. 37] It is found that in two dimensions, the mean-field transition temperature is replaced by a crossover temperature where the correlation length starts to grow exponentially. At sufficiently low temperature, a Kosterlitz-Thouless $\mathrm{O}(2)$ transition should occur, but it is not reproduced by that approach since it is in the $O(n=\infty)$ universality class [38]. Nevertheless, 
the agreement with Monte Carlo calculations is quantitative for both one and two-particle correlation functions over the whole range of parameters accessible by Monte Carlo calculations where it is found that in two dimensions, a pseudogap appears in the single-particle spectral weight [25] as well as in the density of states. [20] Recent dimensional crossover study by Preosti et al. [39] shows that the pseudogap effect is basically absent in three dimensions. In weak to intermediate coupling, the appearance of a pseudogap is traced back to the growing critical pairing fluctuations in the low-temperature renormalized classical regime of the low-dimensional system. With increasing temperature, the spectral weight fills in the pseudogap instead of closing it. Furthermore, the pseudogap appears earlier in the density of states than in the spectral function. It was also noted that the qualitative features found in this study should apply to the d-wave case. In this paper we study in detail spectral properties and pseudogaps in a model Hamiltonian with $\mathrm{d}$-wave pairing symmetry which is more appropriate for high-temperature superconductors.

We consider on a two dimensional square lattice a simple model Hamiltonian which has a superconducting ground state with d-wave symmetry

$$
\begin{aligned}
H & =\sum_{\vec{k}, \sigma} \varepsilon_{\vec{k}} c_{\vec{k}, \sigma}^{+} c_{\vec{k}, \sigma} \\
& +\frac{1}{N} \sum_{\vec{k}, \vec{k}^{\prime}, \vec{q}} V_{\vec{k}, \overrightarrow{k^{\prime}}} c_{\vec{k}, \uparrow}^{+} c_{-\vec{k}+\vec{q}, \downarrow}^{+} c_{-\vec{k}^{\prime}+\vec{q}, \downarrow} c_{\vec{k}^{\prime}, \uparrow}
\end{aligned}
$$

where $\varepsilon_{\vec{k}}=-2 t\left(\cos k_{x}+\cos k_{y}\right)$ and $N$ is the number of lattice sites. We choose $V_{\vec{k}, \vec{k}^{\prime}}$ as a d-wave separable potential given as $V \Pi(\vec{k}) \Pi\left(\vec{k}^{\prime}\right)$ where $\Pi(\vec{k})=$ $\cos k_{x}-\cos k_{y}$, or as $1.5264 \cos 2 \phi=1.5264 \frac{k_{x}^{2}-k_{y}^{2}}{k_{x}^{2}+k_{y}^{2}}$ where $\phi=\arctan \frac{k_{y}}{k_{x}}$. In the real space notation the interaction term may be written as $H_{\mathrm{I}}=V \sum_{i} \Delta_{i}^{+} \Delta_{i}$ where $\Delta_{i}=\sum_{\delta} g(\delta) c_{i, \downarrow} c_{i+\delta, \uparrow} \cdot g(\delta)$ is the Fourier transform of $\Pi(\vec{k})$ given as $g(\delta)=\frac{1}{N} \sum_{\vec{k}} e^{i \vec{k} \cdot \vec{\delta}} \Pi(\vec{k})$. For example, for the following pair structure,

$$
g(\delta)= \begin{cases}1 / 2 & \text { if } \delta=( \pm 1,0) \\ -1 / 2 & \text { if } \delta=(0, \pm 1) \\ 0 & \text { if otherwise }\end{cases}
$$

it leads to $\Pi(\vec{k})=\cos k_{x}-\cos k_{y}$. Obviously $\Pi(\vec{k})=$ $1.5264 \cos 2 \phi$ requires in $g(\delta)$ further neighbors beyond the four nearest neighbors. Here a numerical factor of 1.5264 is introduced to normalize $g(\delta)$ with $\sum_{\delta} g^{2}(\delta)=1$. In terms of $\Delta_{i}$ the d-wave pairing susceptibility is defined as

$$
\chi_{p p}(i, \tau)=\left\langle T_{\tau} \Delta_{i}(\tau) \Delta_{0}^{+}\right\rangle,
$$

where $T_{\tau}$ is the imaginary time ordering operator.
It is well-known that for many strongly correlated electronic models there is no obvious small parameter with which a systematic perturbative approximation can be made. In such a situation, a non-perturbative approach may be a good alternative for at least qualitative understanding of the physics in those strongly correlated model Hamiltonians. In this paper we apply a non-perturbative sum rule approach to the model Hamiltonian (11), which is still not a fully controlled approximation in nature. Because of an extended nature of $\Delta_{i}$ for a d-wave pair and the increasing difficulty of the derivation in the real space representation, here we use the analogy from our previous studies for the repulsive 40,37] and attractive Hubbard models. [36] These studies show the important many-body modification with respect to the standard RPA and T-matrix approaches. The modification comes in two different places. First, the paring susceptibility is calculated through vertex function $U_{p p}$ instead of bare interaction strength $U$, which is constant in our approximation. Second, the pairing fluctuations are coupled to electrons, leading to the self-energy with $U U_{p p}$ form instead of $U^{2}$. In particular, the latter structure is in agreement with the fact that there is no Migdal theorem for this problem, contrary to the case of electron-phonon interactions. And the renormalized interaction constant $U_{p p}$ is determined by the exact sum rule for the pairing susceptibility. By using the sum rule and the renormalized constant $U_{p p}$, we determine in effect the GinzburgLandau parameters due to mode-mode coupling. Thus this approach is similar to the one-loop renormalization group approach within the Gaussian approximation and as a result the Mermin-Wager theorem is formally satisfied in two dimensions. 41] This sum rule approach was systematically compared with the QMC simulations for the repulsive and attractive Hubbard models and the agreement was in a quantitative level both in one- and two-particle functions. We believe that this generic feature of the many-body modification should also carry over to a Hamiltonian with d-wave pairing symmetry. In this modification, the pairing susceptibility and the self-energy can be written in Fourier space in terms of renormalized interaction strength $V_{p p}$

$$
\begin{aligned}
\chi_{p p}(q) & =\frac{\chi_{p p}^{0}(q)}{1+V_{p p} \chi_{p p}^{0}(q)}, \\
\Sigma(k) & =-V V_{p p} \Pi^{2}(\vec{k}) \frac{T}{N} \sum_{q} \chi_{p p}(q) G^{0}(q-k),
\end{aligned}
$$

where the irreducible susceptibility is defined as

$$
\chi_{p p}^{0}(q)=\frac{T}{N} \sum_{k} \Pi^{2}(\vec{k}) G^{0}(q-k) G^{0}(k) .
$$

The above expressions are reduced to the standard Tmatrix approximation when $V_{p p}$ is replaced by bare $V$. We determine this renormalized constant by employing the exact sum rule for the $d$-wave pairing susceptibility 


$$
\begin{aligned}
& \frac{T}{N} \sum_{q} \chi_{p p}(q) e^{-i \nu_{m} 0^{-}} \\
= & \sum_{\delta, \delta^{\prime}} g(\delta) g\left(\delta^{\prime}\right)\left\langle c_{\delta^{\prime}, \downarrow}^{+} c_{\delta, \downarrow} c_{\uparrow}^{+} c_{\uparrow}\right\rangle .
\end{aligned}
$$

Note that the convergence factor is necessary in the sum rule, because away from half-filling $\chi_{p p}\left(i \nu_{m}\right)$ decays like $1 / \nu_{m}$ at large frequencies. In the previous studies for the repulsive and attractive Hubbard models, the righthand side of the sum rule evaluated in the SDW and BCS mean-field ground states, respectively, was found to be in excellent agreement with the QMC values in the intermediate to strong coupling regimes. 42] In this paper the right-hand side of Eq. (5) is also evaluated in the d-wave BCS mean-field ground state

$$
\begin{aligned}
& \frac{1}{N^{2}} \sum_{\vec{k}, \vec{p}, \vec{k}^{\prime}, \vec{p}^{\prime}} \Pi(\vec{k}) \Pi(\vec{p})\left\langle c_{\vec{k}, \downarrow}^{+} c_{\vec{p}, \downarrow} c_{\vec{k}^{\prime}, \uparrow}^{+} c_{\vec{p}^{\prime}, \uparrow}\right\rangle \delta_{\vec{k}+\vec{k}^{\prime}, \vec{p}+\vec{p}^{\prime}} \\
= & {\left[\frac{1}{N} \sum_{\vec{k}} \Pi^{2}(\vec{k}) v^{2}(\vec{k})\right]\left[\frac{1}{N} \sum_{\vec{k}} v^{2}(\vec{k})\right] } \\
+ & {\left[\frac{1}{N} \sum_{\vec{k}} \Pi(\vec{k}) u(\vec{k}) v(\vec{k})\right]^{2} }
\end{aligned}
$$

where

$$
\begin{aligned}
u_{\vec{k}}^{2} & =\frac{1}{2}\left(1+\frac{\varepsilon_{\vec{k}}-\mu}{E_{\vec{k}}}\right), \\
v_{\vec{k}}^{2} & =\frac{1}{2}\left(1-\frac{\varepsilon_{\vec{k}}-\mu}{E_{\vec{k}}}\right), \\
E_{\vec{k}} & =\sqrt{\left(\varepsilon_{\vec{k}}-\mu\right)^{2}+\Delta^{2}(\vec{k})} .
\end{aligned}
$$

Here $\Delta(\vec{k})$ is the d-wave BCS mean-field gap. The chemical potential $\mu$ and the gap $\Delta(\vec{k})$ are determined selfconsistently through the number and gap equations for given $V, T$ and $n$.

Before starting we comment on some differences associated with $\Pi(\vec{k})=\cos k_{x}-\cos k_{y}$ and with $\Pi(\vec{k})=$ $1.5264 \cos 2 \phi$ structure. The interaction Hamiltonian with the first structure depends not only on the angle of $\vec{k}$ but also on its magnitude. Thus the pairing interaction is always strongest at $\vec{k}, \vec{k}^{\prime}=( \pm \pi, 0)$ or $(0, \pm \pi)$, although the noninteracting Fermi surface can be far from these points. As a result two features occur for a particle density far away from half-filling (Detailed calculations were performed but not shown in this paper). First, the peaks associated with the precursor of the Bogoliubov quasiparticles occur asymmetrically with respect to the Fermi energy. Second, the locus of $\vec{k}$ points satisfying $\omega-\varepsilon_{\vec{k}}+\mu-\operatorname{Re} \Sigma(\vec{k}, \omega)=0$ at $\omega=0$ can be substantially different from the noninteracting Fermi surface, thus strongly violating the Luttinger's theorem. For $\Pi(\vec{k})=1.5264 \cos 2 \phi$ which depends only on the angle, however, the above features disappear and the locus of $\vec{k}$ points satisfying the above equation is almost identical with the noninteracting Fermi surface. Near half-filling the differences are negligible. Throughout the calculations, lattice spacing, $\hbar$, and $k_{B}$ are set to be unity, and all energies are measured in unit of $t$. We used a discrete lattice as large as $128 \times 128$ in momentum space and performed the calculations by means of fast Fourier transforms (FFT). Equations (3) and (4i) are computed in terms of Matsubara frequencies and the analytic continuation from Matsubara to real frequencies are made via Pade approximants. 443] In order to detect any spurious features associated with numerical analytical continuation, we performed real frequency calculations in parallel. Except for some spiky features in the real frequency formulation coming from the Lorentzian approximation of the non-interacting Green's function, the two results are almost identical.

We begin by presenting the spectral functions along $(0,0)-(\pi, 0)$ direction for $V=-4 t, n=0.5$ and $T=0.15 t$ in Fig. 1. Throughout the paper the density is fixed at $n=0.5$. The results for other densities are similar. For this parameter, $V_{p p}$ satisfying the sum rule is found to be $-1.87 t$ significantly different from the bare value $(V=-4 t)$. This shows the importance of the mode-mode coupling effect already in the intermediate coupling regime. Let us label wave vectors by $(m \pi / 8,0)$. Below the Fermi wave vector $(m=5)$, the main peak stays below the Fermi energy and the secondary peak grows in strength as $\vec{k}$ approaches $\vec{k}_{F}$. At the Fermi wave vector two peaks reminiscent of the Bogoliubov quasiparticles appear almost symmetrically with respect to the Fermi energy. Since electrons are still in the normal state, this is the precursor of the superconducting gap, namely, a normal state pseudogap. Above the Fermi wave vector, the main peak stays above the Fermi energy and the secondary peak becomes stronger as $\vec{k}$ approaches $\vec{k}_{F}$. This result should be contrasted with that by Engelbrecht et al. [34] In the self-consistent T-matrix approximation with $\Pi(\vec{k})=\cos k_{x}-\cos k_{y}$ form, these authors argued that along $(0,0)-(\pi, 0)$ direction the dominant peak of $A(\vec{k}, \omega)$ never crosses the Fermi energy and bounces back towards the negative frequency. Their overall finding, however, is qualitatively different from our results. In the present calculations, the dominant peak of $A(\vec{k}, \omega)$ eventually passes through the Fermi energy for $\vec{k}$ far above the Fermi wave vector. At the noninteracting Fermi surface our spectral weight is strongly suppressed at the Fermi energy to become a local minimum, while the local minimum of $A(\vec{k}, \omega)$ in their study is located significantly away from the Fermi energy. Presumably this is due to the features associated with $\Pi(\vec{k})=\cos k_{x}-\cos k_{y}$ structure mentioned in the previous paragraph and also due to the self-consistent approximation that does not take the vertex function and the Green's function at the same level of approximation. 44

In Fig. 2 the spectral function is shown along the Fermi 
wave vectors at low temperature $(T=0.15 t)$. The angle $\phi$ is defined as $\arctan \left(k_{y} / k_{x}\right)$ along the noninteracting Fermi surface. At $\phi=0^{\circ}$ the magnitude of the pseudogap is largest and as $\phi$ increases it progressively decreases, leading to strong momentum or angle dependence in the size of the pseudogap. Since along the diagonal directions the pairing interaction vanishes, the pseudogap completely closes and a sharp quasiparticle peak appears at $\phi=45^{\circ}$. In Fig. 3 the magnitude of the pseudogap (circles) is plotted as a function of angle $\phi$ for $T=0.15 t$ along with the ground state gap symmetry $\Delta\left(\phi=0^{\circ}\right) \cos 2 \phi$ (dashed curve). The angle dependence of the normal state pseudogap is well fitted by d-wave symmetry, consistent with ARPES experiments for underdoped cuprates.

At higher temperatures a drastic change is found in the spectral function. For $T=0.225 t$ the pseudogap closes well below $45^{\circ}$, as shown in Fig. A. The local minimum at the Fermi energy disappears at $\phi=31.0^{\circ}\left(26.6^{\circ}\right.$ is just on the crossover). Beyond this angle, the quasiparticlelike peak appears and thereby the Fermi surface is partially restored. We define this angle as critical angle $\phi_{c}$. In Fig. $5 \phi_{c}$ is plotted for different temperatures (open circles). Below $T=0.175 t$ the pseudogap is found everywhere along the Fermi wave vectors except at $\phi=45^{\circ}$, thus the Fermi surface is destroyed everywhere except along the diagonal directions. With increasing temperature (up to $T=0.29 t$ ), however, $\phi_{c}$ becomes smaller than $45^{\circ}$. Thus, the pseudogap region shrinks and at the same time the Fermi surface grows from $\phi=45^{\circ}$ up to the critical angle. Above $T=0.29 t$ the whole Fermi surface is completely restored in spite of some broadening of the spectral function due to interaction as well as finite temperature. This feature is qualitatively consistent with ARPES for underdoped cuprates. [6] We can theoretically calculate the temperature dependence of $\phi_{c}$. By using the Ornstein-Zernike form of the pairing correlation function and taking the classical fluctuations $\left(i q_{n}=0\right)$, [45] the scattering rate at the Fermi energy is found to be proportional to $\Pi^{2}(\vec{k}) \xi / \xi_{T}$, where $\xi$ and $\xi_{T}$ are pairing correlation length and thermal de Broglie wave length defined as $\xi_{T}=v_{F}(\vec{k}) / T$, respectively. For the pseudogap to disappear, the scattering rates should be much smaller than unity $\Pi^{2}(\vec{k}) \xi / \xi_{T} \ll 1$, allowing us to define a critical angle $\xi=v_{F}\left(\phi_{c}\right) / T / \Pi^{2}\left(\phi_{c}\right)$. In Fig. 5 this critical angle is also shown as stars. For the best fit near $0^{\circ}$ angle, a numerical factor of 1.06 multiplies $\xi$. Although a small deviation is found near $45^{\circ}$, the overall magnitude and shape are in reasonable agreement throughout the whole angle. For a d-wave model, the condition for the appearance of a pseudogap in a given momentum depends not only on the anisotropy of the Fermi velocity (which is the only relevant condition in the attractive Hubbard model) but also more importantly on an angle dependent form factor $\Pi^{2}(\vec{k})$.
In Fig. 6 we show the spectral function at the Fermi wave vector and the density of states for different temperatures. As the temperature is increased, the spectral weight starts to fill in the pseudogap and at $T=0.3 t$ the precursor of the superconducting gap completely disappears as shown in Fig. 6(a). At this temperature, however, the pseudogap still persists in the density of states and at higher temperature $(T=0.4 t)$ it finally disappears as shown in Fig. 6(b). Except close to half-filling, the pseudogap appears at higher temperature in the density of states than in the spectral function. Compared with the s-wave case, the density of states is suppressed linearly near the Fermi energy, a reminiscence of $N(\omega) \sim \omega$ in the superconducting state. Both in the spectral function and the density of states, the pseudogap disappears in a manner that the spectral weight fills in the pseudogap instead of closing it with increasing temperature. This feature is also consistent with ARPES for underdoped cuprates. This may suggest that in our approach phase fluctuations (spin-wave type) rather than amplitude fluctuations are mainly responsible for the pseudogap formation, although the present approach includes both. Like in the s-wave case, a pseudogap also appears in the density of states when the characteristic pairing frequency $\nu_{c}$ is equal to or smaller than temperature. This corroborates the origin of the d-wave pseudogap, namely, growing d-wave paring fluctuations in the low temperature renormalized classical regime of the low dimensional system. The calculated $T^{*}$ follows the same trend as the mean-field critical temperature $T_{M F}$. (To be more precise, $T^{*}$ is approximately half of $T_{M F}$ for most of the densities.) As noted in Ref. [26], near a point with high order parameter symmetry (half-filling in that paper), the transition temperature $T_{c}$ decreases while the pseudogap temperature increases along with $T_{M F}$. As a result it leads to a large pseudogap regime, consistent with the phase diagram in the underdoped side of cuprates.

There are several advantages in our formulation. First, the pairing fluctuation sum rule Eq. (5) is exactly satisfied (by construction). Through this sum rule, the Mermin-Wagner theorem is formally fulfilled and the strength of pairing fluctuations is properly constrained within the Gaussian approximation. This latter feature is crucial in our formulation, because an approximate treatment of pairing fluctuations without constraining the strength can easily overestimate or underestimate the magnitude of fluctuations particularly in low dimensional systems. The Mermin-Wagner theorem is also satisfied in some other approaches such as the selfconsistent T-matrix (FLEX) and the "pairing" approximation schemes. Second, there is an exact relation between one-particle (self-energy, Green's function) and two-particle (interaction term) functions: 
$\lim _{\tau \rightarrow 0^{-}} \frac{T}{N} \sum_{\vec{k}, i \omega_{n}} \Sigma\left(\vec{k}, i \omega_{n}\right) G\left(\vec{k}, i \omega_{n}\right) e^{-i \omega_{n} \tau}=V\left\langle\Delta_{i}^{+} \Delta_{i}\right\rangle$,

where here the self-energy includes the Hartree-Fock term. When the interacting Green's function $G\left(\vec{k}, i \omega_{n}\right)$ is replaced by the noninteracting one $G^{0}\left(\vec{k}, i \omega_{n}\right)$ in Eq. (8), it is exactly satisfied. With $G\left(\vec{k}, i \omega_{n}\right)$, the difference between the left- and right-hand sides is less than $6 \%$ for all temperatures studied. In this paper the KosterlitzThouless phase transition 46] and its fluctuation effect near $T_{K T}=T_{c}$ have been neglected, since the present formulation is inadequate to describe the topological nature of the phase in two dimensions and vortex anti-vortex binding-unbinding physics. Since for similar parameters for the attractive Hubbard model the QMC results indicate that $T_{K T} \sim 0.05 t 220$ three times smaller than the lowest temperature in our calculations, we do not expect in the present results any significant influence from vortex phase fluctuations. Our approach is valid for weak to intermediate coupling and for temperature not too deep in the pseudogap regime. At very low temperature, even the Ginzburg-Landau functional form itself may change, for instance, a possible crossover of the dynamical critical exponent from $\mathrm{z}=2$ to other value, 47 and eventually vortex phase fluctuations may come into play.

In summary, we have studied spectral properties and pseudogaps in a model with d-wave pairing symmetry by using a non-perturbative sum rule approach. We applied to this model our previous experience of manybody theory in the repulsive and attractive Hubbard models. The magnitude of the normal state pseudogap shows strong angle dependence well fitted by $\cos 2 \phi$ form at low temperature. With increasing temperature the pseudogap closes at some critical angle $\phi_{c}$ and beyond this angle a single quasiparticle-like peak appears. The resulting Fermi surface is strongly temperature dependent. With increasing temperature, the pseudogap region shrinks and at the same time the Fermi arc grows from $\phi=45^{\circ}$ to $\phi_{c}$. Both in the spectral function and density of states the pseudogap disappears in a manner that the spectral weight fills in the pseudogap instead of closing it with increasing temperature. All these features are qualitatively consistent with ARPES for underdoped cuprates. The pseudogap is caused by growing d-wave critical pairing fluctuations in the low-temperature classical renormalized regime of the low-dimensional system, as in the repulsive and attractive Hubbard models. We argue that although the real critical behaviors and critical exponents are governed by the vortex phase fluctuations close to the $T_{K T}$, the initial growth of pairing fluctuations can be driven by spin-wave phase fluctuations, leading to the normal state pseudogap formation. The behavior of the spin-wave type phase fluctuations belonging to the $O(n=\infty)$ universality class 38 can be qualitatively different in two dimensions (particularly near a point with high order parameter symmetry) from that of the $O(2)$ Kosterlitz-Thouless vortex phase fluctuations.

The author would like to thank A. M. Tremblay for numerous help and discussions throughout the work. This work was supported by a grant from the Natural Sciences and Engineering Research Council (NSERC) of Canada and the Fonds pour la formation de Chercheurs et d'Aide à la Recherche (FCAR) of the Québec government.

[1] B. G. Levi, Phys. Today, 46, 17 (1993); ibid. 49, 19 (1996).

[2] Z.-X. Shen, D. S. Dessau, B. O. Wells, D. M. King, W. E. Spicer, A. J. Arko, D. Marshall, L. W. Lombardo, A. Kapitulnik, P. Dickinson, S. Doniach, J. DiCarlo, T. Loeser, and C. H. Park, Phys. Rev. Lett. 70, 1553 (1993).

[3] D. S. Marshall, D. S. Dessau, A. G. Loeser, C-H. Park, A. Y. Matsuura, J. N. Eckstein, I. Bozovic, P. Fournier, A. Kapitulnik, W. E. Spicer, and Z.-X. Shen, Phys. Rev. Lett. 76, 4841 (1996).

[4] H. Ding, T. Yokoya, J. C. Campuzano, T. Takahashi, M. Randeria, M. R. Norman, T. Mochiku, K. Kadowaki, and J. Giapintzakis, Nature 382, 51 (1996).

[5] A. G. Loeser, Z. -X. Shen, D. S. Dessau, D. S. Marshall, C. H. Park, P. Fournier, A. Kapitulnik, Science 273, 325 (1996).

[6] H. Ding et al., J. Phys. Chem. Solids 59, 1888 (1998).

[7] J. W. Loram, K. A. Mirza, J. R. Cooper, and W. Y. Liang, Phys. Rev. Lett. 71, 1740 (1993); Physica (Amsterdam) 235C-240C, 134 (1994).

[8] Ch. Renner, B. Revaz, J. -Y. Genoud, K. Kadowaki, and ØFischer, Phys. Rev. Lett. 80, 149 (1998).

[9] M. Takigawa, P. C. Hammel, R. H. Heffner, and Z. Fisk, Phys. Rev. B 43, 247 (1991).

[10] J. Orenstein, G. A. Thomas, A. J. Millis, S. L. Cooper, D. H. Rapkine, T. Timusk, L. F. Schneemeyer, and J. V. Waszczak, Phys. Rev. B 42, 6342 (1990); L. D. Rotter, Z. Schlesinger, R. T. Collins, F. Holtzberg, C. Field, U. W. Welp, G. W. Crabtree, J. Z. Liu, Y. Fang, K. G. Vandervoort, and S. Fleshler, Phys. Rev. Lett. 67, 2741 (1991); C. C. Homes and T. Timusk, R. Liang, D. A. Bonn, and W. N. Hardy, Phys. Rev. Lett. 71, 1645 (1993).

[11] P. W. Anderson, Science 235, 1196 (1987).

[12] Baskaran, Z. Zou, and P. W. Anderson, Solid State Commun. 69, 973 (1987); P. W. Anderson, G. Baskaran, Z. Zou, and T. Hsu, Phys. Rev. Lett. 58, 2790 (1987); A. E. Ruckenstein, P. J. Hirshfield, and J. Appel, Phys. Rev. B 36, 857 (1987); G. Kotliar and J. Liu, Phys. Rev. B 385142 (1988); Y. Suzumura, Y. Hasegawa, and H. Fukuyama, J. Phys. Soc. Jpn. 57, 401 (1988).

[13] P. A. Lee and X. G. Wen, Phys. Rev. Lett. 78, 4111 (1997).

[14] S. Doniach and M. Inui, Phys. Rev. B 41, 6668 (1990).

[15] V. J. Emery and S. A. Kivelson, Nature 374, 434 (1995).

[16] M. Franz and A. J. Millis, Phys. Rev. B 58, 14572 (1998); M. Capezzali and H. Beck, Phsica B 259-261, 
501 (1998); H. J. Kwon and A. T. Dorsey, Phys. Rev. B 59, 6438 (1999); V. P. Gusynin, V. M. Loktev, and S. G. Sharapov, JETP 88685 (1999).

[17] Z. X. Shen and J. R. Schrieffer, Phys. Rev. Lett. 78, 1771 (1997).

[18] J. Schmalian, D. Pines, and B. Stojkovic, Phys. Rev. Lett. 80, 3839 (1998); A. V. Chubukov, condmat/9709221 (unpublished).

[19] R. T. Scalettar, E. Y. Loh, J. E. Gubernatis, A. Moreo, S. R. White, D. J. Scalapino, R. L. Sugar, and E. Dagotto, Phys. Rev. Lett. 62, 1407 (1989).

[20] A. Moreo and D. J. Scalapino, Phys. Rev. Lett. 66, 946 (1991); A. Moreo, D. J. Scalapino, and S. R. White, Phys. Rev. B 45, 7544 (1992).

[21] M. Randeria, N. Trivedi, A. Moreo, and R. T. Scalettar, Phys. Rev. Lett. 69, 2001 (1992).

[22] F. F. Assaad, W. Hanke, and D. J. Scalapino, Phys. Rev. Lett. 71, 1915 (1993); Phys. Rev. B 49, 4327 (1994).

[23] N. Trivedi and M. Randeria, Phys. Rev. Lett. 75, 312 (1995).

[24] J. M. Singer, M. H. Pedersen, T. Schneider, H. Beck, and H.-G. Matuttis, Phys. Rev. B 54, 1286 (1996); J. M. Singer, T. Schneider, and M. H. Pedersen, Eur. Phys. J. B 1, 1 (1998); J. M. Singer, T. Schneider, and P. F. Meier, Eur. Phys. J. B 7, 37 (1999).

[25] Y. M. Vilk, S. Allen, H. Touchette, S. Moukouri, L. Chen, and A.-M. S. Tremblay, J. Phys. Chem. Solids, 59, 1873 (1998).

[26] S. Allen, H. Touchette, S. Moukouri, Y. M. Vilk, and A.-M. S. Tremblay, Phys. Rev. Lett. 83, 4128 (1999).

[27] B. Janko, J. Maly, and K. Levin, Phys. Rev. B 56, R 11 407 (1997); Q. Chen, I. Kosztin, B. Janko, and K. Levin, Phys. Rev. Lett. 81, 4708 (1998).

[28] J. W. Serene, Phys. Rev. B 40, 10873 (1989).

[29] R. Frésard, B. Glasser, and P. Wölfle, J. Phys. Cond. Matt. 4, 8565 (1992).

[30] R. Micnas, M. H. Pedersen, S. Schafroth, T. Schneider, J. J. Rodríguez-Núñez, and H. Beck, Phys. Rev. B 52, 16223 (1995).

[31] J. J. Deisz, D. W. Hess, and J. W. Serene, Phys. Rev. Lett. 80, 373 (1998).

[32] Bumsoo Kyung, E. G. Klepfish, and P. E. Kornilovitch, Phys. Rev. Lett. 80, 3109 (1998).

[33] P. E. Kornilovitch and Bumsoo Kyung, J. Phys. Cond. Matter, 11, 741 (1999).

[34] J. R. Engelbrecht, A. Nazarenko, M. Randeria, and E. Dagotto, Phys. Rev. B 57, 13406 (1998).

[35] T. Hotta, M. Mayr, and E. Dagotto, cond-mat/9903208 (unpublished).

[36] Bumsoo Kyung, S. Allen, and A. -M. S. Tremblay (unpublished).

[37] Y. Vilk and A. M. Tremblay, J. Phys. I (France) 7, 1309 (1997).

[38] A. M. Dare, Y. M. Vilk and A. M. Tremblay, Phys. Rev. B 53, 14236 (1996).

[39] G. Preosti, Y. M. Vilk, and M. R. Norman, Phys. Rev. B 59, 1474 (1999).

[40] Y. M. Vilk, Liang Chen, and A. M. Tremblay, Phys. Rev. B 49, 13267 (1994).

[41] N. D. Mermin and H. Wagner, Phys. Rev. Lett. 17, 1133 (1966).
[42] In the weak coupling limit $(|U| \leq t-2 t)$, however, the right-hand side (double occupancy in the Hubbard model) evaluated in the BCS ground state is underestimated compared with the exact value. This is in contrast to a general belief that in the weak coupling limit the BCS ground state is exact. Through an interpolation scheme, a weak coupling case may be covered as well. In this paper the intermediate coupling regime is studied.

[43] H. J. Vidberg and J. W. Serene, J. Low Temp. Phys. 29, 179 (1977).

[44] There are some exceptional cases where a pseudogap behavior is found in $A(\vec{k}, \omega)$ even in the self-consistent Tmatrix approach, when the $\vec{q}=0$ pairing mode is separated from the particle-particle scattering continuum (local preformed-pair). 32, 33.35 This condition is met for a large coupling strength and more importantly for a small particle density.

[45] Y. M. Vilk and A. M. Tremblay, Europhys. Lett. 33, 159 (1996).

[46] J. M. Kosterlitz and D. J. Thouless, J. Phys. C 6, 1181 (1973).

[47] S. Sachdev, A. V. Chubukov, and A. Sokol, Phys. Rev. B 51, 14874 (1995). 


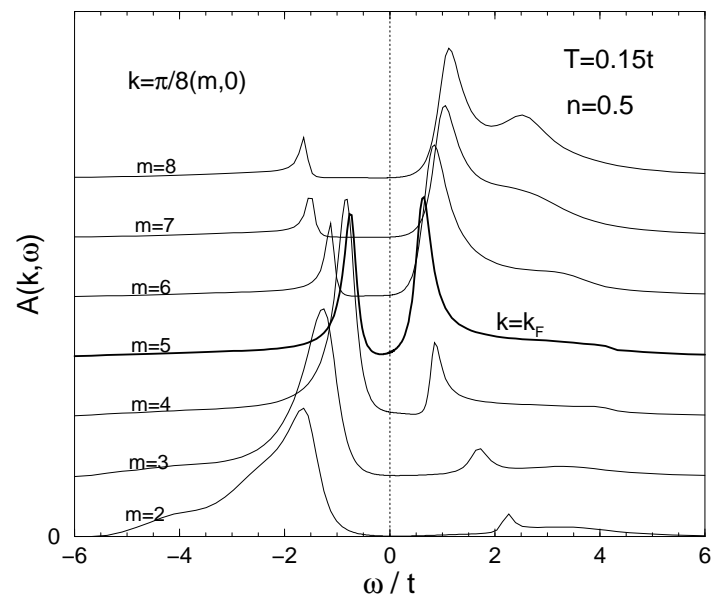

FIG. 1. Spectral function along the line $(0,0)-(\pi, 0)$ for $V=-4, n=0.5$ and $T=0.15$. The figures for $m=3-8$ are shifted vertically by 0.25 . $m=5$ corresponds to the noninteracting Fermi surface.

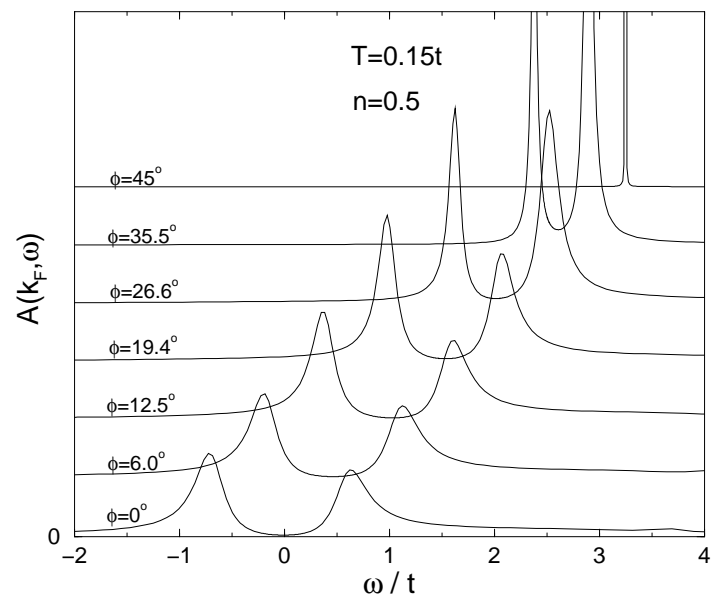

FIG. 2. Spectral function along the noninteracting Fermi surface for $V=-4, n=0.5$ and $T=0.15$. The angle $\phi$ is defined as $\arctan \left(k_{y} / k_{x}\right)$ along the noninteracting Fermi surface. The figures for $\phi>0^{\circ}$ are shifted vertically and horizontally both by 0.5 .

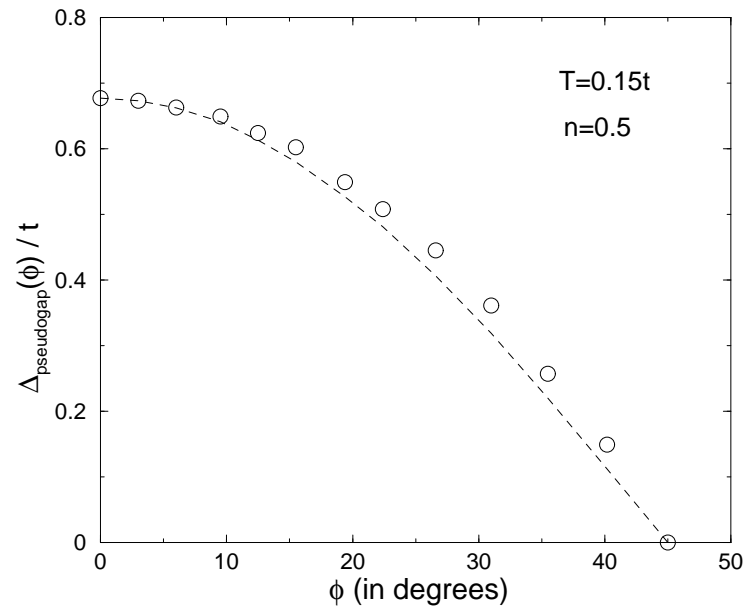

FIG. 3. The magnitude of the pseudogap for $V=-4$, $n=0.5$ and $T=0.150$ (open circles). The angle $\phi$ is defined as $\arctan \left(k_{y} / k_{x}\right)$ along the noninteracting Fermi surface. The dashed curve is $\Delta\left(\phi=0^{\circ}\right) \cos 2 \phi$.

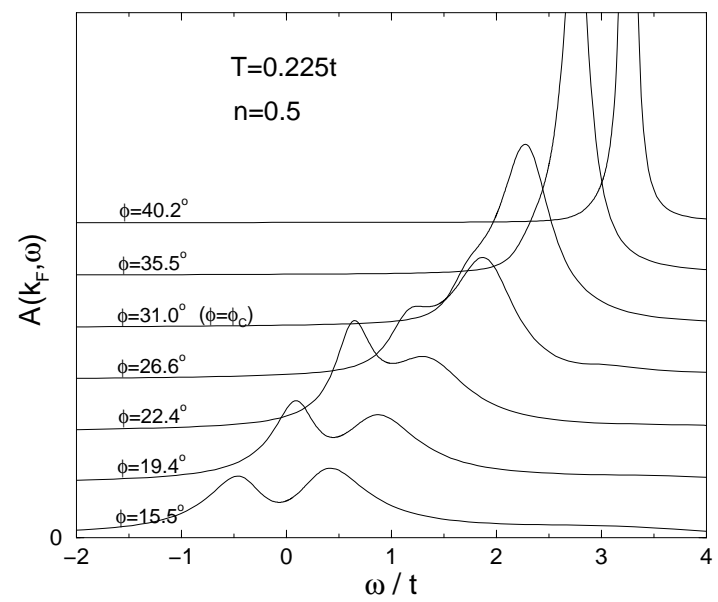

FIG. 4. Spectral function along the noninteracting Fermi surface for $V=-4, n=0.5$ and $T=0.225$. The angle $\phi$ is defined as $\arctan \left(k_{y} / k_{x}\right)$ along the noninteracting Fermi surface. The figures for $\phi>15.5^{\circ}$ are shifted vertically and horizontally by 0.25 and 0.5 , respectively. 


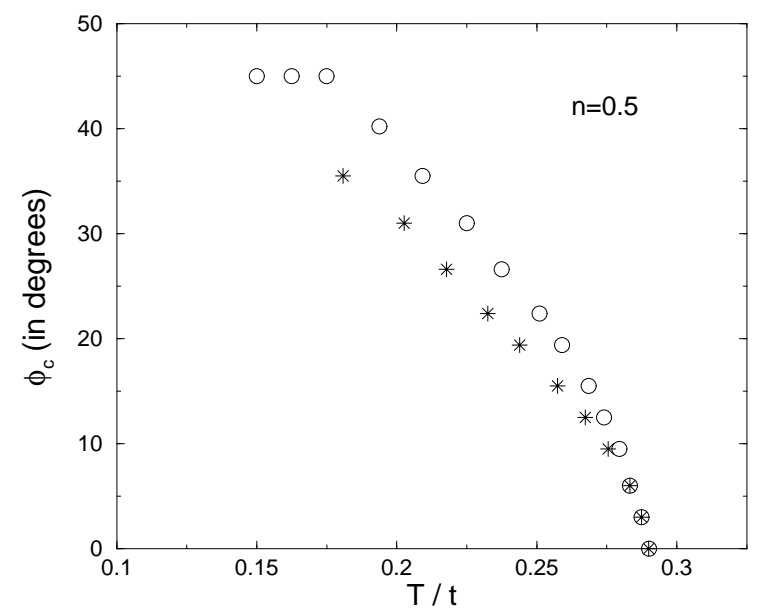

FIG. 5. Critical angle $\phi_{c}$ as a function of temperature for $V=-4, n=0.5$ (open circles). The stars are the critical angle estimated by the expression given in the text.
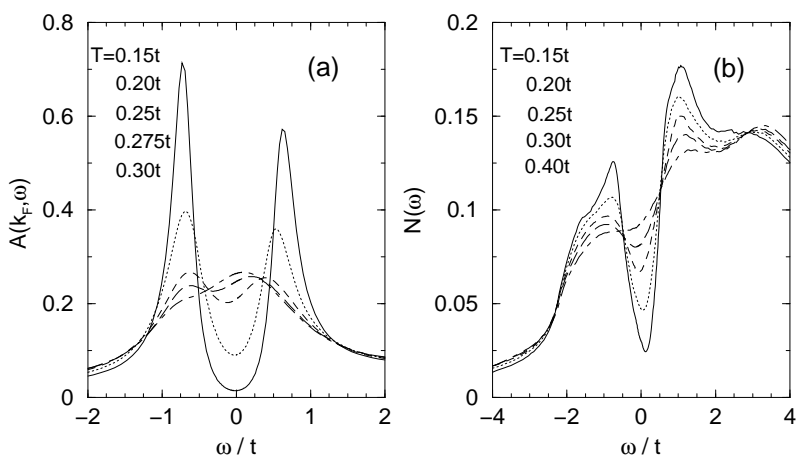

FIG. 6. (a) The spectral function at the noninteracting Fermi surface and (b) the density of states for $V=-4$ and $n=0.5$ at different temperatures. The solid, dotted, dashed, long-dashed, and dot-dashed curves correspond to temperatures from top to bottom. 